\title{
Ultrasound Elastography as A Motion Estimation Problem
}

\author{
Marwan H. Hussein, Yasser M. Kadah \\ Systems \& Biomedical Engineering Department \\ Cairo University \\ Giza, Egypt
}

\begin{abstract}
We study the feasibility of processing B-mode images instead of RF data to generate ultrasound elastography displacement fields. We use the exhaustive search (ES) algorithm which is a basic block matching algorithm from the video compression domain. We also apply two modifications to ES that can enhance both speed and detectability. Quantitative measurements of accuracy and runtime are presented. Results and potential future work are discussed.
\end{abstract}

\section{INTRODUCTION}

Ultrasound (US) static elastography has been a potential medical imaging topic for research \& application for about 20 years [1], [2], [3]. It can be noticed from the literature that US elastography development continued to depend in its majority on RF processing [1]. However, access to RF data of commercial US machines is not always available. Access to B-mode images is more available. So, developing elastography techniques that can process B-mode images instead of RF data can ease integrating elastography as a third party module to commercial machines.

In this work, we study the feasibility (quality and runtime) of applying motion estimation techniques (from the video compression domain) on B-mode images to generate displacement fields.

\section{MATERIALS \& METHODS}

For testing purposes we used the same phantom data in Rivaz et al. as the input to our algorithm [4]. Both B-mode images (pre \& postcompression) were cropped to the area containing the lesion. Then, both images were input to the motion estimation algorithm.

\section{A. Block Matching Algorithms - Exhaustive Search}

We used one of the block matching algorithms (BMAs) (commonly used in video compression domain) to estimate the displacement field of the precompression image.

For our application (US elastography), it's desirable to get the displacement vector for every pixel to get a dense motion field (for accuracy), so we used overlapping blocks with spatial difference 1 and chose Mean Absolute Difference (MAD) as the cost function. The search parameter was set to 5 . There are various techniques for block matching which differ in accuracy \& speed [5], but we chose to start with the most computationally expensive, yet most accurate, algorithm - the exhaustive search (ES), to assess the quality of motion fields generated using BMAs with B-mode image.
After applying ES to pre \& postcompression images the displacement field is generated.

\section{B. First Modification of ES (upper-only search)}

After compressing the imaged tissue by the probe, the distance between any locus in the tissue and the probe gets smaller. Consequently, every locus appears in an upper location in the postcompression image than its original location in the precompression image. Utilizing this, and because we were using the precompression image as the reference image, we applied a modification to the ES algorithm in which we limit the search range vertically to the upper half of the search window. We expected this modification to enhance the quality of the displacement field (besides enhancing speed definitely) as it should reduce false positives. We will be referring to this modified ES technique as single-modification ES (SMES) for the rest of the paper.

\section{Second Modification of ES (limiting search in horizontal direction)}

Another property of elastography images is that the majority of the displacement should be in the vertical (axial) direction due to the nature of the compression (which is axial). This let us apply an additional modification to the SMES algorithm in which we limited the search range in the horizontal (lateral) direction to be less than vertical search (we set horizontal search to 2). Again we expected this to further reduce false positives in addition to enhancing speed. We will be referring to SMES with the second modification as double-modified ES (DMES) for the rest of the paper.

\section{Smooth and Subpixel Displacement Estimation}

Two main problems need to be addressed in US elastography techniques even those operating on RF data. One problem is that the displacement field should be naturally smooth (i.e. continuous) because the tissue is a continuum [4]. The other problem is that both vertical \& horizontal displacements generated using simple correlationlike techniques are integer displacements, so estimation of subpixel displacement may be required [4]. Both problems cause resultant displacement fields to appear noisy (and pixelated).

Both problems should also exist in our case (operating on B-mode images instead of RF data). We tried to alleviate the first problem by applying a median filter $(7 * 7)$ to both vertical \& horizontal displacement fields resulting from DMES algorithm to remove false peaks. We then tried to 
solve the second problem by upsampling both vertical \& horizontal displacement fields by spline interpolation.

\section{RESULTS}

We chose two unitless measures for quantitative assessment of motion fields. The first one is the signal-tonoise ratio (SNR) and the second measure is the normalized Mean Square Error percentage $\left(M S E_{\text {norm }}\right)$. It should be noted that we didn't have access to a gold standard for the displacement field of the phantom in order to compare our resultant field to it, so we instead calculated $M S E_{\text {norm }}$ between the motion-compensated image and the true postcompression image.

The runtime of the motion estimation block $\left(t_{M E}\right)$ (on a $2.2 \mathrm{GHz}$ processor), the $S N R$ for the vertical displacement field $\left(S N R_{V}\right)$ and the horizontal displacement fields $\left(S N R_{H}\right)$, and the $M S E_{\text {norm }}$ between the motion-compensated image and the true postcompression image are shown in Table I for the three ES configurations and the DMES configuration with median filtration \& spline upsampling.

\section{DISCUSSION}

The $M S E_{\text {norm }}$ values in Table 1 are generally low (which is desired). It's also shown in Table I that the runtime is reduced in SMES and further reduced in DMES than basic ES while $S N R_{V}$ and $S N R_{H}$ values do not vary significantly.

However, the resulting displacement fields are noisy and cannot be interpreted by the human eye. Also, $S N R_{V} \&$ $S N R_{H}$ values are overall lower than Rose's criterion [6] despite any enhancement caused by modifying basic ES. ES detectability with US elastography images may not be the same as in the case of photographic images due to some differences between the nature of images in both cases. Also, B-mode images have less information than RF data, so the main problems addressed during RF processing should be addressed during B-mode processing with more emphasis. We believe that applying more powerful solutions to the two main problems mentioned before in the Methods section (displacement continuity and subpixel displacement estimation) will lead to significant improvement of current results.

It's also shown in Table. 1 that despite the enhancements in the motion estimation runtime by modifying the basic ES, the runtime is still not suitable for real-time applications (to be integrated in a commercial US machine).

TABLE I. QuANTITATIVE MEASURES FOR THE DIFFERENT ES CONFIGURATIONS

\begin{tabular}{|c|c|c|c|c|}
\hline Measure & $\begin{array}{c}\text { Basic } \\
\text { ES }\end{array}$ & SMES & DMES & $\begin{array}{c}\text { DMES with } \\
\text { median } \\
\text { applied \& } \\
\text { upsampling }\end{array}$ \\
\hline$t_{M E}(\mathrm{~s})$ & 174.7 & 91.5 & 45.9 & 45.9 \\
\hline$S N R_{V}$ & 1.87 & 1.27 & 1.22 & 1.35 \\
\hline$S N R_{H}$ & 1.7 & 1.7 & 1.86 & 1.7 \\
\hline$M S E_{\text {norm }}(\%)$ & 0.0644 & 0.1333 & 0.4248 & -- \\
\hline
\end{tabular}

\section{CONCLUSION \& FUTURE WORK}

We presented an initial feasibility study of generating displacement fields by processing B-mode images with the basic block matching algorithm ES and its two modifications. Results and discussion indicate that further problems need to be addressed to achieve reliable quality. Some of these problems are already addressed in implemented RF processing techniques.

Many points are potential to future work. Enhancing subpixel displacement estimation and displacement continuity can lead to better displacement fields. Also, contrast enhancement of B-mode images can lead to better detectability. Making the search range and block size functions of US imaging parameters (like sampling rate) and applied compression can help the automation of the search. If these trials show better elastographic quality then runtime can be enhanced by applying more optimized BMAs (like Adaptive Rood Pattern Search (ARPS)). Another runtime enhancement that can be applied to ES is making a parallel implementation of the algorithm to utilize multiple CPUs or graphics processing units (GPUs).

\section{REFERENCES}

[1] K. Parker, M. Doyley, and D. Rubens, "Imaging the elastic properties of tissue: the 20 year perspective," Physics in Medicine and Biology, vol. 56, p. R1, 2010.

[2] P. R. Hoskins, K. Martin, and A. Thrush, Diagnostic ultrasound: physics and equipment: Cambridge University Press, 2010.

[3] J. Ophir, I. Cespedes, H. Ponnekanti, Y. Yazdi, and X. Li, "Elastography: a quantitative method for imaging the elasticity of biological tissues," Ultrasonic imaging, vol. 13, pp. 111-134, 1991.

[4] H. Rivaz, E. Boctor, P. Foroughi, R. Zellars, G. Fichtinger, and G. Hager, "Ultrasound elastography: a dynamic programming approach," Medical Imaging, IEEE Transactions on, vol. 27, pp. 13731377, 2008.

[5] A. Barjatya, "Block matching algorithms for motion estimation," ed: unpublished.

[6] J. T. Bushberg, J. A. Seibert, E. M. Leidholdt, and J. M. Boone, The essential physics of medical imaging: Lippincott Williams \& Wilkins, 2001. 